\title{
Direct Experimental Simulation of the Yang-Baxter Equation
}

\author{
Chao Zheng, ${ }^{1,2}$ Jun-lin Li, ${ }^{1} \quad$ Si-yu Song, ${ }^{1}$ and Gui Lu Long, ${ }^{1,2, *}$ \\ ${ }^{1}$ State Key Laboratory of Low-Dimensional Quantum Physics and Department of Physics, \\ Tsinghua University, Beijing 100084, P.R. China \\ ${ }^{2}$ Tsinghua National Laboratory for Information Science and Technology, Beijing 100084, P. R. China
}

compiled: October 17, 2018

\begin{abstract}
Introduced in the field of many-body statistical mechanics, Yang-Baxter equation has become an important tool in a variety fields of physics. In this work, we report the first direct experimental simulation of the Yang-Baxter equation using linear quantum optics. The equality between the two sides of the Yang-Baxter equation in two dimension has been demonstrated directly, and the spectral parameter transformation in the Yang-Baxter equation is explicitly confirmed.
\end{abstract}

OCIS codes: $\quad$ 030.0030, 270.0270.

\section{Introduction}

Yang-Baxter equation (YBE) was originated from solving the repulsive $\delta$ interaction problem in one-dimension of $N$ particles [1, 2], and problems of statistical models on lattices [3 5]. Today, Yang-Baxter equation has become an important tool in physics, and has many applications in variety of areas of physics, for instance in quantum field theory, statistical mechanics, and group theories [3-15]. It can be applied in completely integrable statistical models to find the solutions by means of the nested Bethe ansatz [15]. Recently it turns up gradually that Yang-Baxter equation is naturally linked to a hot area of frontier research, the quantum information and computing 16, 17. It is found that the Yang-Baxter equation is closely related to quantum entangled states [18, 19], the braiding operations in the Yang-Baxter equation are universal quantum gates $20-$ 24]. Yang-Baxter equation attracts much attention in recent years and is being studied in the context of quantum correlation and entanglement, and topological quantum computing intensively 25,32$]$.

Due to its importance, the experimental verification of Yang-Baxter equation has been pursued all along. Notably, an experimental verification was carried out by Tennant et al in 1995 33, 34]. Tennant et al measured the spectrum of Heisenberg spin-half chain, and the experimental result appeared to agree with the calculation based on the Yang-Baxter equation. Recently, the density profile of 1-dimensional wires was measured and it agreed well with the theoretical calculations based on Yang's solvable model [35]. However, these experiments

* Corresponding author: gllong@tsinghua.edu.cn are indirect verifications of the Yang-Baxter equation because the Yang-Baxter equation provides only a sufficient condition for the spectrum or profile, or the observed profile is only a necessary condition for the YangBaxter equation and it does not guarantee the validity of the Yang-Baxter equation. Thus the direct verification of the Yang-Baxter equation is still an open question [36]. Direct experimental verification of the YangBaxter equation requires not only the verification of the equality of the left-hand and the right-hand sides of the equation, but also the transformation relation between the spectral parameters in the Yang-Baxter equation, the Lorentz-like transformation.

In this paper, we report the first direct experimental simulation of the Yang-Baxter equation using quantum optics. The fundamental principles of the present simulation was established in 2008 by $\mathrm{Hu}$, Xue and $\mathrm{Ge}$ [36]. Hu, Xue and Ge gave an explicit optical realization of the Yang-Baxter equation. By the use of the Temperley-Lieb algebra, they made a remarkable reduction that obtained a Yang-Baxter equation with dimension 2, the minimum dimensional Yang-Baxter equation so far. This makes it possible to be implemented in quantum optics with current technology. In our experiment, we experimentally implemented the $\mathrm{Hu}$-Xue-Ge scheme and demonstrated the validity of the Yang-Baxter equation using linear quantum optical components such as beamsplitters, half-wave plates, quarter wave plates, and etc. The equality of the two sides of the Yang-Baxter equation is directly verified. In particular, the Lorentzlike transformation in the spectral parameters of the Yang-Baxter equation is experimentally demonstrated for the first time. The present experiment completes the first direct experimental simulation of the Yang-Baxter equation. 


\section{Theoretical framework}

The Yang-Baxter equation reads,

$$
\breve{R}_{12}(u) \breve{R}_{23}\left(u_{23}\right) \breve{R}_{12}(v)=\breve{R}_{23}(v) \breve{R}_{12}\left(u_{23}\right) \breve{R}_{23}(u),
$$

where $u$ and $v$ are spectral parameters, and $\beta^{-1}=i c(c$ is the light speed in vacuum), and

$$
u_{23}=\frac{u+v}{1+\beta u v}
$$

is the Lorentz-like transformation relation of the spectral parameters. The $N^{2} \times N^{2}$ dimension matrix $\breve{R}$ acts on the tensor product space $V \otimes V$ of two $N$ dimensional spaces, and is the two-particle scattering matrix depending on the relative rapidity $\tanh ^{-1}(\beta u)$. When $\beta u=1, \breve{R}=b$ which is a braid matrix, and the Yang-Baxter equation reduces to the braid relation $b_{12} b_{23} b_{12}=b_{23} b_{12} b_{23}$. This equation implies the scattering of particles 1 and 2, followed by scattering of particles 2 and 3, and then scattering of particles 1 and 2, is equal to the scattering of particles 2 and 3, followed by scattering of particles 1 and 2, and then scattering of particles 2 and 3, when they satisfy the Yang-Baxter equation with suitable spectral parameters.

Yang-Baxter equation is an abstract equation and the quantities in the equation may have different meanings in different problem. For instance, it has been found recently that the braid matrix and the Yang-Baxter equation are connected to entangled quantum states [21]. The Bell-basis entangled states in four-dimension can be obtained by applying braid operation that satisfies the Yang-Baxter equation on the computational basis. Here the matrix in the Yang-Baxter equation becomes a transformation that transforms the computational basis to the Bell-basis states. There have been active studies in this direction, interested readers can refer to Ref. [36] and references therein for more details.

The scheme used in our experiment was proposed by $\mathrm{Hu}$, Xue and Ge recently [36]. The Yang-Baxter equation with the minimum nontrivial dimension is in four dimension. In this case, $\breve{R}$ becomes a $4 \times 4$ matrix. In principle, it can be simulated directly by means of quantum optics, and $\mathrm{Hu}$, Xue and Ge gave an explicit optical realization. However, such a realization requires many controlled NOT gates whose realization is of very low efficiency in linear quantum optics [37, 38] that its feasibility using current technology is illusive. A further simplification was made by the use of the Temperley-Lieb algebra [39], and the Yang-Baxter equation with minimal dimension was reduced further to 2 . This makes it feasible to implement in quantum optics with current technology.

The 2-dimensional Yang-Baxter equation is expressed as,

$$
A(u) B\left(\frac{u+v}{1+\beta^{2} u v}\right) A(v)=B(v) A\left(\frac{u+v}{1+\beta^{2} u v}\right) B(u),
$$

where

$$
A(u)=\rho(u)\left(\begin{array}{cc}
\frac{1+\beta^{2} u^{2}+2 i \epsilon \beta u}{1+\beta^{2} u^{2}-2 i \epsilon \beta u} & 0 \\
0 & 1
\end{array}\right)
$$

and

$$
B(u)=\frac{\rho(u)}{1+\beta^{2} u^{2}-2 i \epsilon \beta u}\left(\begin{array}{cc}
1+\beta^{2} u^{2} & 2 i \epsilon \beta u \\
2 i \epsilon \beta u & 1+\beta^{2} u^{2}
\end{array}\right),
$$

and $\rho(u)$ is a normalization factor and $\epsilon= \pm 1$.

For convenience in optical realization, $A$ and $B$ are represented as functions of optical parameter $\theta$, the angle between the optical axes of an optical device and the vertical direction. The two sets of parameters are related by using the following transformation

$$
\frac{1+\beta^{2} u^{2}+2 i \epsilon \beta u}{1+\beta^{2} u^{2}-2 i \epsilon \beta u} \equiv e^{-2 i \theta}
$$

and

$$
\rho(u) \equiv e^{i \theta}
$$

$A(u)$ and $B(u)$ then become simple matrices in two dimensions

$$
A(\theta)=\left(\begin{array}{cc}
e^{-i \theta} & 0 \\
0 & e^{i \theta}
\end{array}\right)
$$

and

$$
B(\theta)=\left(\begin{array}{cc}
\cos \theta & -i \sin \theta \\
-i \sin \theta & \cos \theta
\end{array}\right) .
$$

The Yang-Baxter equation in Eq. (3) can be re-written as

$$
A\left(\theta_{1}\right) B\left(\theta_{2}\right) A\left(\theta_{3}\right)=B\left(\theta_{3}\right) A\left(\theta_{2}\right) B\left(\theta_{1}\right) .
$$

The three parameters in the Yang-Baxter equation $\theta_{1}, \theta_{2}$ and $\theta_{3}$ are not independent, and they are related through the following equation

$$
\left(e^{-2 i \theta_{2}}+1\right)\left[i-\sec \left(\theta_{1}-\theta_{3}\right) \sin \left(\theta_{1}+\theta_{3}\right)\right]=2 i .
$$

Using this relation, we can transform the Lorentz-like relation in spectral parameters into a relation in the optical angle parameters,

$$
\theta_{2}=\arctan \left(\frac{\sin \left(\theta_{1}+\theta_{3}\right)}{\cos \left(\theta_{1}-\theta_{3}\right)}\right)
$$

We use the photon polarization qubit in our experiment. A general elliptically polarized photon state $|\psi\rangle$ can be written as

$$
|\psi\rangle=\alpha|\uparrow\rangle+i \beta|\leftrightarrow\rangle,
$$

where $\alpha$ and $\beta$ are real and satisfy $|\alpha|^{2}+|\beta|^{2}=1$. Without loss of generality, we assume $\alpha$ is real and positive. The sign of the $\beta$ specifies the handedness of the circular 
polarized photon. $|\uparrow\rangle$ and $|\leftrightarrow\rangle$ are basis states of the vertical and horizontal polarization respectively. State $|\psi\rangle$ is measured directly in the experiment.

The operations of $A(\theta)$ and $B(\theta)$ can be realized by series of quarter-wave plates (QWP) and half-wave plates (HWP), whose effects are equivalent to two elements of an $\mathrm{SU}(2)$ transformation group [40] as shown in Fig. 1,

(a)
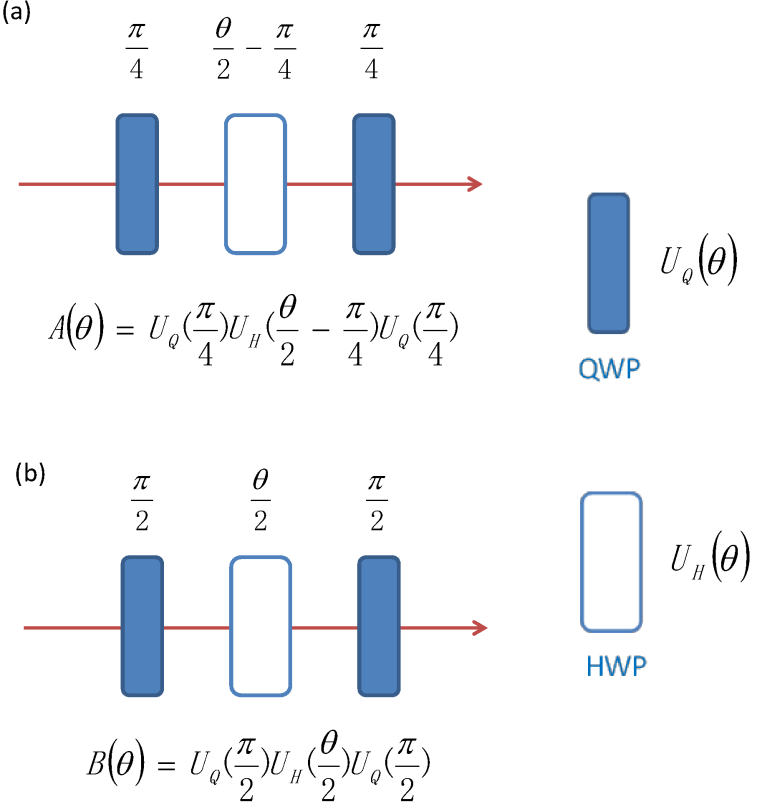

Fig. 1. (Color online) Realization of operations (a) $A(\theta)$ and $B(\theta)$ by optical elements. $U_{Q}(\theta)$ and $U_{H}(\theta)$ are the matrices of QWP and HWP, respectively, and $\theta$ is the angle between the optical device axes and the vertical direction.

$$
U_{Q}(\theta)=\frac{1}{\sqrt{2}}\left(\begin{array}{cc}
1-i \cos (2 \theta) & -i \sin (2 \theta) \\
-i \sin (2 \theta) & 1+i \cos (2 \theta)
\end{array}\right)
$$

and

$$
U_{H}(\theta)=U_{Q}^{2}(\theta)=-i\left(\begin{array}{cc}
\cos (2 \theta) & \sin (2 \theta) \\
\sin (2 \theta) & -\cos (2 \theta)
\end{array}\right),
$$

where $\theta$ is the angle between the optical axis of QWP or HWP and the vertical direction. Thus, $A(\theta)$ and $B(\theta)$ can be simulated by series of QWP and HWP, i.e.

$$
A(\theta)=U_{Q}\left(\frac{\pi}{4}\right) U_{H}\left(-\frac{\pi}{4}+\frac{\theta}{2}\right) U_{Q}\left(\frac{\pi}{4}\right)
$$

and

$$
B(\theta)=U_{Q}\left(\frac{\pi}{2}\right) U_{H}\left(\frac{\theta}{2}\right) U_{Q}\left(\frac{\pi}{2}\right),
$$

respectively. The two sides of the Yang-Baxter equation are simulated by two series of wave plates as illustrated in Fig. 2 , (a)
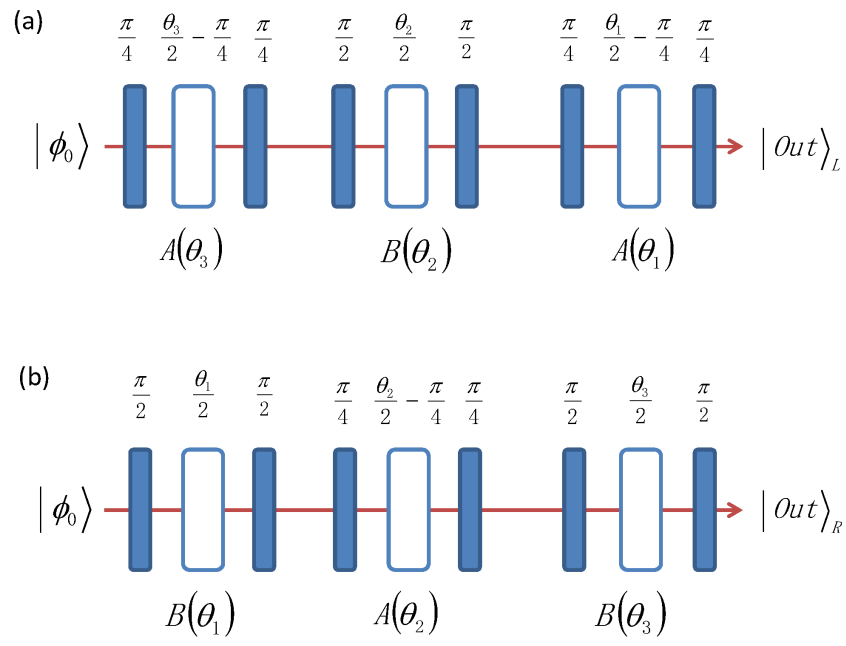

Fig. 2. (Color online) Optical realization of the (a) LHS and RHS of Yang-Baxter equation. The angles of these QWPs (filled) and HWPs (empty) must satisfy the Lorentz-like relation given in Eq. (12).

The qubit state after the transformation of the LHS of the Yang-Baxter equation is denoted as,

$$
\left|\psi_{\text {out }}\right\rangle_{L}=\alpha_{L}|\uparrow\rangle_{L}+i \beta_{L}|\leftrightarrow\rangle_{L}
$$

and the qubit state after the processing of the RHS of the Yang-Baxter equation can be expressed as

$$
\left|\psi_{\text {out }}\right\rangle_{R}=\alpha_{R}|\uparrow\rangle_{R}+i \beta_{R}|\leftrightarrow\rangle_{R}
$$

To check the equality of these two final output states, we define the fidelity

$$
\mathrm{C}_{\mathrm{YBE}}=\left|{ }_{L}\left\langle\psi_{\text {out }} \mid \psi_{\text {out }}\right\rangle_{R}\right| \text {, }
$$

which is the absolute value of the overlap of the two states. Fidelity $\mathrm{C}_{\mathrm{YBE}}$ is a good measure of the validity of the Yang-Baxter equation. If $\mathrm{C}_{\mathrm{YBE}}$ equals to 1 , the two sides of the Yang-Baxter equation is equal. Otherwise it is not valid. In the real experiment, it should be 1 within statistical errors, and independent of the input state.

\section{Experimental method and results}

The experimental setup is explicated in Fig. 3. A HeNe laser with center frequency $632.8 \mathrm{~nm}$, drawn in the left part of Fig. 3, is used to generate sequences of photons with certain polarization state. The input state can be prepared at arbitrary linearly or elliptically polarized photon states conveniently by using either a HWP or a QWP following the PBS and an attenuator. In the experiments, the intensity of the light source are attenuated to a weak level that well approximate the single photon sources. Then the photons go through a series of 
optical components which simulate either the left-handside or the right-hand-side of the Yang-Baxter equation, which is indicated in the middle part of Fig. 3. The right part of Fig. [3 is used to measure the polarized state of the photon output state after going through corresponding Yang-Baxter equation transformation.

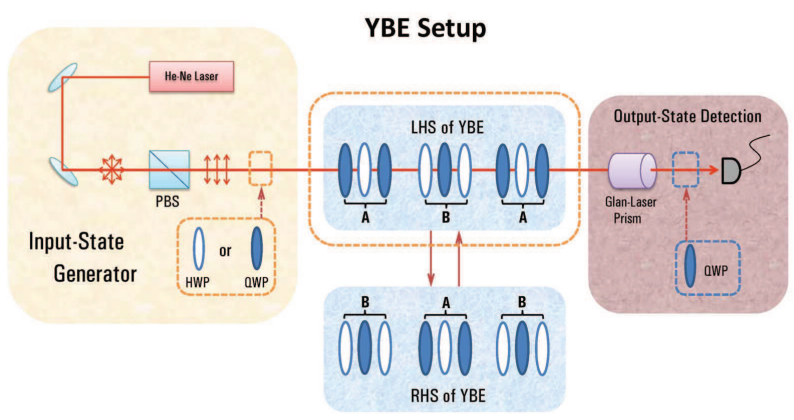

Fig. 3. (Color online) Experimental setup. The left part generates a horizontally polarized state, which is then transformed into a desired input state with arbitrary linearly or elliptically polarization by using a HWP or QWP, respectively, following the PBS. The middle part is the left-hand side or right-hand side of Yang-Baxter equation consists of series of wave plates. The right part, containing a Glan-Laser prism and a single photon detector, is used to detect the polarized state of the output state. A QWP may be inserted to determine the handedness of an elliptically polarized photon.

\section{A. Verification of Equality of the Yang-Baxter equation}

Groups of angles $\theta_{1}, \theta_{2}$ and $\theta_{3}$ are selected and experimented. The fidelity $\mathrm{C}_{\mathrm{YBE}}$ parameter is measured for each group of angles. The experimental results prove the Yang-Baxter equation very well. For illustration purpose, we set the two angles $\theta_{1}$ and $\theta_{3}$ at values $56^{\circ}$ and $23^{\circ}$ respectively in the following. We then vary $\theta_{2}$ from $0^{\circ}$ to $180^{\circ}$ and obtain $\mathrm{C}_{\mathrm{YBE}}$ for each $\theta_{2}$.

The curve of $\mathrm{C}_{\mathrm{YBE}}$ versus $\theta_{2}$ for the input state $|\uparrow\rangle$ is presented in Fig. 4. $\mathrm{C}_{\mathrm{YBE}}$ reaches its maximum value $0.9997 \pm 0.0237$ when $\theta_{2}=49.49^{\circ}$, which is equal 1 within statistical error. From this one can see that for an arbitrary values of angles, $\theta_{1}, \theta_{2}$ and $\theta_{3}, A\left(\theta_{3}\right) B\left(\theta_{2}\right) A\left(\theta_{1}\right)$ and $B\left(\theta_{1}\right) A\left(\theta_{2}\right) B\left(\theta_{3}\right)$ are usually not equal. Only when the three angles satisfy the Lorentz-like relation, Eq. (12), namely, they satisfy the Yang-Baxter equation, the operations are equal. This clearly verified the validity of the Yang-Baxter equation.

For the input state $|\leftrightarrow\rangle$, identical results, within statistical error, as those for input state $|\uparrow\rangle$ are obtained.

If an input state is an arbitrarily polarized state, say, $(0.7071-0.5417 i)|\uparrow\rangle-0.4545 i|\leftrightarrow\rangle$, the fidelity $\mathrm{C}_{\mathrm{YBE}}$ versus $\theta_{2}$ curve is depicted in Fig. 5. One can see that $\mathrm{C}_{\text {YBE }}$ reaches the maximum value 1 within statistical error when $\theta_{2}=49.49^{\circ}$, which accords with the theoretical value determined by Eq. (12); while other $\theta_{2}$ values do not make $\mathrm{C}_{\mathrm{YBE}}$ equal to 1 , which implies $A\left(\theta_{3}\right) B\left(\theta_{2}\right) A\left(\theta_{1}\right)$ and $B\left(\theta_{1}\right) A\left(\theta_{2}\right) B\left(\theta_{3}\right)$ are not equal when they do not satisfy Eq. (12). In this case, the Yang-Baxter equation is not satisfied. Therefore, the two operations are equal only when the Yang-Baxter equation condition is met. It firmly demonstrates the validity of the Yang-Baxter equation.

As seen already above, $A\left(\theta_{3}\right) B\left(\theta_{2}\right) A\left(\theta_{1}\right)$ and $B\left(\theta_{1}\right) A\left(\theta_{2}\right) B\left(\theta_{3}\right)$ are not identical for arbitrary sets of $\theta_{i}, i=1,2,3$. It is clearly manifested again in the difference between the two curves in Fig. 4 and Fig. 5, namely the fidelity $\mathrm{C}_{\mathrm{YBE}}$ differs for operations $A\left(\theta_{3}\right) B\left(\theta_{2}\right) A\left(\theta_{1}\right)$ and $B\left(\theta_{1}\right) A\left(\theta_{2}\right) B\left(\theta_{3}\right)$. They transform the same input state to different output state for general values of $\theta_{2}$. The Yang-Baxter equation corresponds to a specific setting, that is, $\mathrm{C}_{\mathrm{YBE}}$ is 1 if three angles satisfy Eq. (12). In this particular values of $\theta_{1}$ and $\theta_{3}$ displayed in Fig. [4 and 5, $\theta_{2}=49.49^{\circ}$ satisfies the Yang-Baxter equation, as they are clearly demonstrated in Fig. 4 and Fig. 5. The transformations representing the two sides of the Yang-Baxter equation transform any input state into the same output state, clearly validating the equality of the Yang-Baxter equation in the experimental results.

\section{B. Verification for the necessity of Lorentz-like transformation}

We have verified the sufficiency of the Lorentz-like transformation of parameters $u$ and $v$ in Eq. (3) until now. In term of the parameters $\theta_{1}, \theta_{2}$ and $\theta_{3}$, the transformation is expressed in Eq. (12). However, It can not be excluded that other groups of $\theta_{1}, \theta_{2}$ and $\theta_{3}$, which don't satisfy the Lorentz-like transformation, also validate the Yang-Baxter equation just by the former experimental verification. It is essential to verify that the Lorentzlike transformation of parameters $u$ and $v$ in Eq. (3) is the necessary condition to validate the Yang-Baxter equation. In other words, we need to find out all the relations of $\theta_{1}, \theta_{2}$ and $\theta_{3}$ that make Yang-Baxter equation valid.

Converting it to experiment, we need to record all groups of $\theta_{1}, \theta_{2}$ and $\theta_{3}$ when the fidelity $\mathrm{C}_{\mathrm{YBE}}$ reaches 1 within statistical error. To achieve this, we fixed $\theta_{3}$ first. For each fixed $\theta_{3}$, we kept $\theta_{2}$ and tune $\theta_{1}$ from 0 to $\pi$ continually to find out all pairs of $\theta_{1}$ and $\theta_{2}$ that make the fidelity $\mathrm{C}_{\mathrm{YBE}}$ almost 1 (here we choose the lower bound of $\mathrm{C}_{\mathrm{YBE}}$ as 0.9995 ). The experimental results show that all groups of $\theta_{1}, \theta_{2}$ and $\theta_{3}$ satisfy Eq. (12) when $\mathrm{C}_{\mathrm{YBE}}$ attains 1 within statistical error, i.e. the original spectral parameters $u$ and $v$ satisfy the Lorentzlike transformation.

To illustrate this, we show three series of our experimental results, i.e. three curves of $\theta_{2}$ versus $\theta_{1}$ for the corresponding three fixed $\theta_{3}$ when $\mathrm{C}_{\mathrm{YBE}}$ equals to 1 within the statistical error. We draw the relation curves of $\theta_{2}$ versus $\theta_{1}$ for different values of $\theta_{3}$ while keeping $\mathrm{C}_{\text {YBE }}>0.9995$ in Fig. 6, where $\theta_{3}$ are fixed at $32^{\circ}$ (blue dots), $56^{\circ}$ (green squares), and $146^{\circ}$ (red triangles) respectively. The theoretical figures are also shown in Fig. 
6 (lines), from which one can see that the experimental results agree with the theoretical prediction very well. The rich structure of the Lorentz-like relation is well confirmed in the experiment.

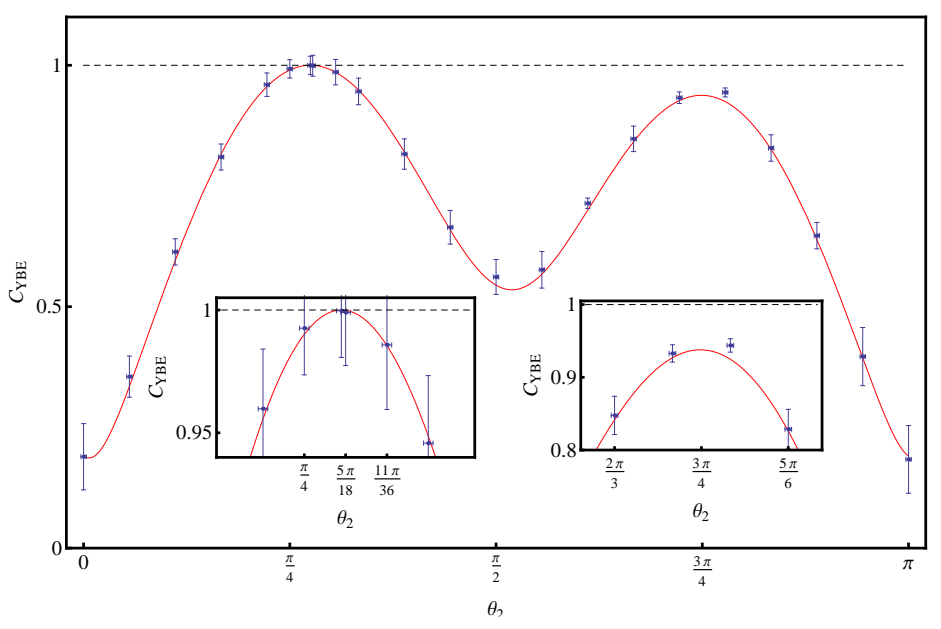

Fig. 4. (Color online) The curve of $\mathrm{C}_{\mathrm{YBE}}$ versus $\theta_{2}$ where $\theta_{1}$ and $\theta_{3}$ are kept fixed at $56^{\circ}$ and $23^{\circ}$, respectively. The dots are the experimental data while the line is the theoretical curve. The input state is $|\uparrow\rangle$, the vertical polarization state. When the input state is chosen as $|\leftrightarrow\rangle$, the horizontally polarized state, both experimental and theoretical results are identical to those for the vertical polarization input state.

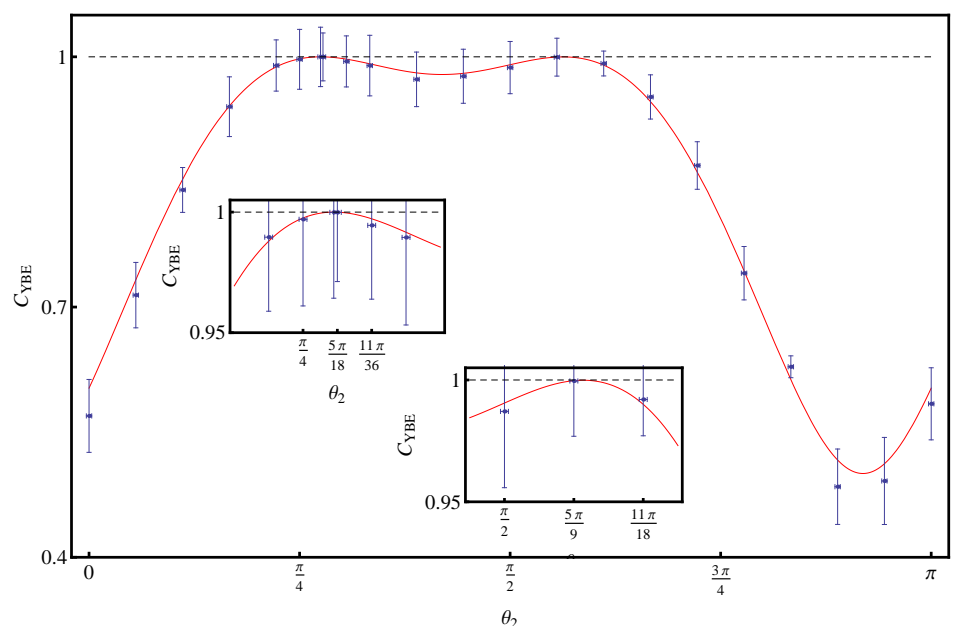

Fig. 5. (Color online) The curve of $\mathrm{C}_{\mathrm{YBE}}$ versus $\theta_{2}$ where $\theta_{1}$ and $\theta_{3}$ are kept fixed at $56^{\circ}$ and $23^{\circ}$, respectively. The dots are the experimental data while the line is the theoretical curve. The input state is an elliptically polarized state with the form $(0.7071-0.5417 i)|\uparrow\rangle-0.4545 i|\leftrightarrow\rangle$. $\mathrm{C}_{\mathrm{YBE}}$ reaches $0.9999 \pm 0.0356$ when $\theta_{2}=49.49^{\circ}$.

\section{Summary, review and outlook}

The Yang-Baxter equation is directly verified experimentally using linear quantum optics devices for the first time from the following two aspects. On one hand, the

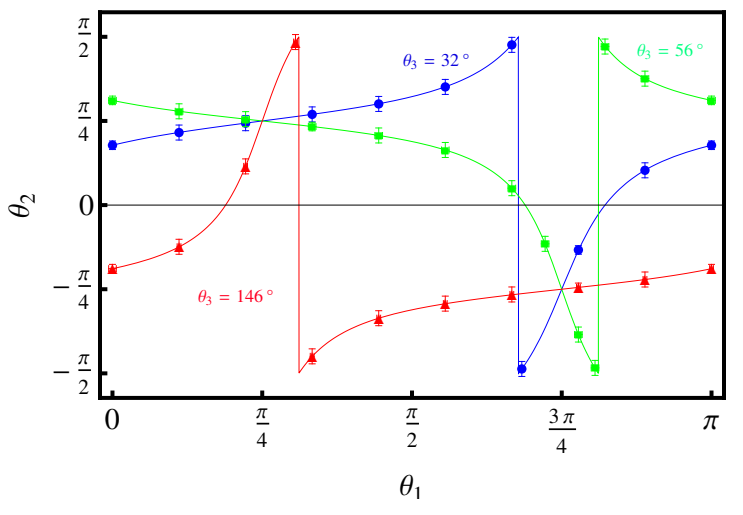

Fig. 6. (Color online) $\theta_{2}$ versus $\theta_{1}$ curve while fixing $\theta_{3}$. The blue dots, the green squares and the red triangles are the experimental data for $\theta_{3}=32^{\circ}, 56^{\circ}$, and $146^{\circ}$, while the lines are the corresponding theoretical results.

experiment proved the equality between the two sides of Yang-Baxter equation if the parameters $\theta_{1}, \theta_{2}$ and $\theta_{3}$ satisfy Eq. (12), which is to $\theta$ 's what Lorentz-like transformation is to the original spectral parameter $u$ and $v$. It means that the validity of Yang-Baxter equation is guaranteed sufficiently when the spectral parameters satisfy the Lorentz-like transformation. On the other hand, we verified that it is also the necessary condition for the validity of Yang-Baxter equation to make the spectral parameters satisfy the Lorentz-like transformation. We recorded all groups of the parameters $\theta_{1}, \theta_{2}$ and $\theta_{3}$ that make the fidelity be 1 within the statistical error, and found out that each group satisfies Eq. (12). In this process, it is fully presented again that the beautiful structure of the Lorentz-like transformation of the spectral parameters.

Two issues remain open for further studies for higher dimensional Yang-Baxter equation. One important issue is the role of entanglement in the Yang-Baxter equation. In the present experiment, no entanglement is involved. In higher dimensions, the operations in the Yang-Baxter equation will inevitably bring in quantum entanglement. It will be an interesting and significant subject for future study, and consequently the entangling power of the operations in Yang-Baxter equation, namely the operations in either sides of the Yang-Baxter equation emerge naturally also an important topic. The effect of Lorentz-like transformation of the Yang-Baxter equation is mythical. It drives two independent operators to become identical as seen from this 2-dimensional quantum system. In higher dimensions, the operations will be more complex and entanglement also comes into play. The role of the transformation relation will be tested and studied in more detail and in wider aspects.

Discovered from solving problems in many-body systems and statistical models in the middle of the last century, variety of contexts of Yang-Baxter equation were revealed and it has been applied to many different area, such as quantum field theory, statistical mechan- 
ics, group theory, and etc. Now, Yang-Baxter equation is playing an important role in quantum information science which is a thriving area of frontier research. Using the relation between Bell basis and Yang-Baxter equation enables the investigation of quantum entanglement, and the relation between anyon and Yang-Baxter equation entails exploring topological quantum computing. Many interesting applications of the Yang-Baxter equation lies ahead. Yang-Baxter equation not only deserves a direct verification, like this work, but also merits scientists' continued investigation.

\section{Acknowledgments}

This work was supported by the National Natural Science Foundation of China (Grant No. 10874 098,11175094), the National Basic Research Program of China (2009CB929402, 2011CB9216002).

\section{References}

[1] C. N. Yang, "Some exact results for the many-body problem in one dimension with repulsive delta-function interaction," Phys. Rev. Lett. 19, 1312 (1967).

[2] C. N. Yang, "S matrix for the one-dimensional N-body problem with repulsive or attractive $\delta$-function interaction," Phys. Rev. 168, 1920 (1968).

[3] R. J. Baxter, Exactly Solved Models in Statistical Mechanics (Academic, 1982).

[4] R. J. Baxter, "Partition function of the Eight-Vertex lattice model," Ann. Phys. 70, 193-228 (1972).

[5] Yang-Baxter Equation in Integrable Systems, edited by M. Jimbo (World Scientific, 1990).

[6] I. B. Frenkel and N. Jing, "Vertex representations of quantum affine algebras," Proc. Natl. Acad. Sci. USA 85, 9373-9377 (1988).

[7] M. Gerstenhaber and S. D. Schack, "Bialgebra cohomology, deformations, and quantum groups," Proc. Natl. Acad. Sci. USA 87, 478-481 (1990).

[8] C. N. Yang and M. L. Ge, Braid Group, Knot Theory and Statistical Mechanics, 2nd ed (World Scientific, 1994).

[9] S. C. Billey, "Kostant polynomials and the cohomology ring for G/B," Proc. Natl. Acad. Sci. USA 94, 29-32 (1997).

[10] C. P. Sun, "The differential realization of new solutions for Yang-Baxter equation", Chin. Sci. Bull. 37, 379-379 (1992).

[11] P. Gui, "Another solution of Yang-Baxter equation on set and 'metahomomorphisms on groups"', Chin. Sci. Bull. 42, 1852-1855 (1997).

[12] H. X. Chen, "Cpcycle deformations, braided monoidal categories and quasitriangularity", Chin. Sci. Bull. 44, 510-513 (1999).

[13] D. P. Hou, and C. M. Bai, "J-dendriform algebras", Front. Math. China, 7, 29-49 (2012)

[14] J. Dubail, J. L. Jacobsen, and H. Saleur, "Exact Solution of the Anisotropic Special Transition in the O(n) Model in Two Dimensions", Phys. Rev. Lett. 103, 145701 (2009).

[15] D. C. Mattis, The Many-Body Problem (World Scientific, 1993).

[16] M. A. Nielsen and I. L. Chuang, Quantum Computa- tion and Quantum Information (Cambridge University Press, 2000).

[17] S. S. Li, G. L. Long, F. S. Bai, S. L. Feng, and H. Z. Zheng, "Quantum Computing," Proc. Natl. Acad. Sci. USA 98, 11847-11848 (2001).

[18] Y. Zhang and M. L. Ge, "GHZ states, almost-complex structure and Yang-Baxter equation," Quantum Inf. Process 6, 363-379 (2007).

[19] J. L. Chen, K. Xue, and M. L. Ge, "Braiding transformation, entanglement swapping, and Berry phase in entanglement space," Phys. Rev. A 76, 042324 (2007).

[20] H. A. Dye, "Unitary solutions to the Yang-Baxter equation in dimension four," Quantum Inf. Process 2, 117152 (2003).

[21] L. H. Kauffman and S. J. Lomonaco Jr, "Braiding operators are universal quantum gates," New J. Phys. 6, 134 (2004).

[22] Y. Zhang, L. H. Kauffman, and M. L. Ge, "Universal quantum gate, Yang-Baxterization and Hamiltonian," Int. J. Quantum Inf. 3, 669-678 (2005).

[23] J. M. Franko, E. C. Rowell, and Z. Wang, "Extraspecial 2-groups and images of braid group representations," Journal of Knot Theory and Its Ramifications 15, 413427 (2006).

[24] E. C. Rowell, Y. Zhang, Y. S. Wu, and M. L. Ge, "Extraspecial two-groups, generalized Yang-Baxter equations and braiding quantum gates," Quantum Inf. Comput. 10, 0685-0702 (2010).

[25] A. Y. Kitaev, "Fault-tolerant quantum computation by anyons," Ann. Phys. 303, 2-30 (2003).

[26] M. H. Freedman, A. Y. Kitaev, and Z. H. Wang, "Simulation of topological field theories by quantum computers," Comm. Math. Phys. 227, 587-603 (2002).

[27] E. Ardonne and K. Schoutens, "Wavefunctions for topological quantum registers," Ann Phys 322, 201-235 (2007).

[28] A. Feiguin, S. Trebst, A. W. W. Ludwig, M. Troyer, A. Kitaev, Z. Wang, and M. H. Freedman, "Interacting anyons in topological quantum liquids: The golden chain," Phys. Rev. Lett. 98, 160409 (2007).

[29] C. Nayak, S. H. Simon, A. Stern, M. Freedman, and S. D. Sarma, "Non-Abelian anyons and topological quantum computation," Rev. Mod. Phys. 80, 1083-1159 (2008).

[30] K. Hikami, "Skein theory and topological quantum registers: Braiding matrices and topological entanglement entropy of non-Abelian quantum Hall states," Ann. Phys. 323, 1729-1769 (2008).

[31] S. Bose and V. Korepin, "Quantum gates between flying qubits via spin-independent scattering," http://arxiv.org/abs/1106.2329v1

[32] X. C. Yao, T. X. Wang, H. Z. Chen, W. B. Gao, A. G. Fowler, R. Raussendorf, Z. B. Chen, N. L. Liu, C. Y. Lu, Y. J. Deng, Y. A. Chen, and J. W. Pan, "Experimental demonstration of topological error correction," Nature 482, 489-494 (2012).

[33] D. A. Tennant, T. G. Perring, R. A. Cowley, and S. E. Nagler, "Unbound spinons in the $\mathrm{S}=1 / 2$ antiferromagnetic chain $\mathrm{KCuF}_{3}$," Phys. Rev. Lett. 70, 4003-4006 (1993).

[34] D. A. Tennant, R. A. Cowley, S. E. Nagler, and A. M. Tsvelik, "Measurement of the spin-excitation continuum in one-dimensional $\mathrm{KCuF}_{3}$ using neutron scattering," Phys. Rev. B 52, 13368-13380 (1995). 
[35] Y. A. Liao, A. S. C. Rittner, T. Paprotta, W. Li, G. B. Partridge, R. G. Hulet, S. K. Baur, and E. J. Mueller, "Spin-imbalance in a one-dimensional Fermi gas," Nature 467, 567-569 (2010).

[36] S. W. Hu, K. Xue, and M. L. Ge, "Optical simulation of the Yang-Baxter equation," Phys. Rev. A 78, 022319 (2008).

[37] P. Kok, W. J. Munro, K. Nemoto, T. C. Ralph, J. P. Dowling, and G. J. Milburn, "Linear optical quantum computing with photonic qubits," Rev. Mod. Phys. 79, 135-174 (2007).

[38] J. L. O' Brien, G. J. Pryde, A. G. White, T. C. Ralph, and D. Branning, "Demonstration of an all-optical quantum controlled-NOT gate," Nature 426, 264-267 (2003).

[39] H. N. V. Temperley, and E. H. Lieb, "Relations between the 'percolation' and 'colouring' problem and other graph-theoretical problems associated with regular planar lattices: Some exact results for the 'percolation' problem," Proc. R. Soc. London, Ser. A 322, 251-280 (1971).

[40] J. Lu, L. Zhou, and L. M. Kuang, "Linear optics implementation for quantum game with two players," Phys. Lett. A 330, 48-53 (2004). 